María Luisa Saavedra García* Jaime Loé Uribe ***

Universidad Nacional Autónoma de México Ciudad de México, México

Recibido: 20 de agosto de 2016

Concepto de evaluación: 25 de mayo de 2018

Aprobado: 24 de junio de 2018

Artículo de investigación (c) 2018 Universidad Católica de Colombia. Facultad de Ciencias Económicas y Administrativas. Todos los derechos reservados

* Doctora en Administración, Docente-investigadora, Universidad Nacional Autónoma de México, Ciudad de México, México. Correo electrónico: Isaavedra@fca.unam.mx. https://orcid.org/0000-0002-32971157.

** Magíster en Finanzas, Docenteinvestigador de la Fundación México-Estados Unidos para la Ciencia (FUMEC). Correo electrónico: jloe@fumec.org.https:// orcid.org/0000-0002-2215-177X.

\section{Flujo de efectivo para las pymes: una propuesta para los sectores automotor y de tecnologías de la información en México"}

\section{RESUMEN}

La administración del efectivo resulta fundamental para la sobrevivencia de las pymes; por ello, en este artículo se propone una herramienta para que los micro y pequeños empresarios del sector automotor y de tecnologías de la información puedan llevar un control del efectivo de sus empresas y de este modo eviten el riesgo de quedarse sin liquidez. Para este fin se utilizó el método de caso múltiple. Los principales hallazgos de este trabajo determinan que la herramienta financiera propuesta constituye un recurso estratégico y útil para la toma de decisiones en la administración del ciclo de conversión de efectivo de las pymes.

Palabras clave: ciclo de conversión de efectivo, flujo de efectivo, pymes, sector automotor, sector TI.

JEL: G39, L62, L86, M21

\section{Cash flow for SMEs: A proposal for the automotive and IT sectors in Mexico}

\section{ABSTRACT}

Cash management is essential for the survival of SMEs; therefore, this work aims to propose a tool for micro and small entrepreneurs in the automotive and IT sectors to keep track of cash in their businesses and to avoid the risk of running out of liquidity. The multiple case method was used for this purpose. The findings of this study evidence that the proposed financial tool is a strategic resource that provides support for decision making in the management of the cash conversion cycle of SMEs.

\footnotetext{
* Este artículo es resultado de un trabajo de colaboración realizado por la Facultad de Contaduría y Administración de la Universidad Nacional Autónoma de México (UNAM) y FUMEC México, en el marco del proyecto de investigación "Herramientas de planeación para la pyme", coordinado por la Dra. María Luisa Saavedra García.
} 
Keywords: SMEs, cash flow, cash conversion cycle, automotive sector, IT sector.

\section{Fluxo de caixa para as PMEs: uma proposta para os setores automotivo e de tecnologias da informação no México}

\section{RESUMO}

A administração do fluxo de caixa é fundamental para a sobrevivência das PMEs; por isso, neste artigo, propõe-se uma ferramenta para que os micro e pequenos empresários do setor automotivo e de tecnologias da informação possam levar um controle do fluxo de caixa de suas empresas e, desse modo, evitem o risco de ficarem sem liquidez. Para isso, utilizou-se o método de caso múltiplo. Os principais achados deste trabalho determinam que a ferramenta financeira proposta constitui um recurso estratégico e útil para a tomada de decisões na administração do ciclo de conversão de caixa das PMEs.

Palavras-chave: ciclo de conversão de caixa, fluxo de caixa, PMEs, setor automotivo, setor TI. 


\section{INTRODUCCIÓN}

Entre las dificultades más importantes de las pymes en Latinoamérica se encuentra la falta de acceso a fuentes de financiamiento, problema que va de la mano de la falta de liquidez que enfrentan los empresarios de estas empresas, pues, al tener recursos escasos, muestran un bajo poder de negociación frente a acreedores y bancos al momento de solicitar crédito, ya sea en especie o en efectivo; en consecuencia, les queda poco margen de maniobra para contar con una liquidez que les permita solventar sus operaciones diarias. Tal problemática es corroborada por Ahmad y Raza (2012), cuyos hallazgos evidenciaron una correlación negativa entre el ciclo de conversión de efectivo y el tamaño de la entidad medido en términos del total de activos. De ahí la importancia de realizar esta investigación, que culmina con una propuesta metodológica para que el empresario cuente con una herramienta de control y planeación del flujo de efectivo; herramienta que ha sido diseñada mediante una intervención en las empresas, con el fin de conocer sus necesidades reales y proponer un instrumento acorde con sus necesidades específicas.

Para tal efecto, la pregunta central que busca resolverse en este artículo es la siguiente: ¿cuál es la metodología de flujo de efectivo que las empresas pymes de México de los sectores automotor y de tecnologías de la información (TI) deben usar para desarrollar su estrategia de planeación de crecimiento e inversión, en función de poder conocer sus necesidades de dinero a corto y mediano plazo?

La investigación es un estudio de caso múltiple y descriptiva, ya que pretende examinar el tema de flujo de efectivo en pymes de los sectores automotor y $\mathrm{TI}$, para luego generar datos. Se pretende lograr una descripción de la situación de las pymes en lo relacionado con el flujo de efectivo, con el fin de identificar la necesidad de información a este respecto en empresas de estas características. Con ello también se busca generar una propuesta de metodología de análisis estratégico de flujo de efectivo que sirva como herramienta especializada de análisis para empresas mexicanas.
El alcance del modelo se refiere únicamente al manejo del efectivo, su control y su impacto en la toma de decisiones respecto de los excedentes o faltantes. Asimismo, será de utilidad no solo para las empresas analizadas en esta investigación, sino también para todo tipo de empresas micro o pequeñas que tengan interés en implementar este modelo y adaptarlo a sus necesidades específicas, dado que es muy comprensible su generación y uso.

\section{MARCO TEÓRICO}

\section{Caracterización de las pymes en México}

La importancia de las pymes en México radica en que representan el $99,80 \%$ de todo el tejido empresarial, generan el $70 \%$ del empleo y aportan el $50 \%$ del producto interno bruto (PIB). De acuerdo con la última estratificación empresarial oficial realizada por la Secretaría de Economía (2009), las pymes se clasifican como se muestra en la tabla 1.

El efecto compuesto de número de trabajadores y monto de ventas anuales se utiliza con el fin de que las empresas de este sector empresarial no queden rezagadas frente a las empresas de mayor tamaño, al participar en los fondos y programas de fomento.

\section{Las pymes del sector automotor}

Uno de los sectores primordiales para el progreso industrial de México es el automotor; el país es uno de los 10 principales productores de automóviles, camiones, partes y componentes en el mundo. En 2008 la producción de este sector fue superior a 2 millones de unidades, un $4 \%$ más en comparación con el año anterior. Para 2010 se produjeron 2,27 millones de unidades, y se mostró incluso más crecimiento en 2011, cuando alcanzó 2,55 millones de unidades; en 2012 alcanzó 2,88 millones de unidades, en tanto en 2013 avanzó a 2,97 millones de unidades. En este último año, el sector representó el $2,6 \%$ del PIB nacional y el $15 \%$ del PIB manufacturero. 
Tabla 1.

Clasificación empresarial de las pymes en México

\begin{tabular}{|c|c|c|c|c|}
\hline Tamaño & Sector & $\begin{array}{c}\text { Rango de número de } \\
\text { trabajadores }\end{array}$ & $\begin{array}{c}\text { Rango de monto de ventas } \\
\text { anuales (mdp) }\end{array}$ & $\begin{array}{c}\text { Tope máximo } \\
\text { combinado }\end{array}$ \\
\hline Micro & Todas & Hasta 10 & Hasta \$4 & 4,6 \\
\hline \multirow{3}{*}{ Pequeña } & Comercio & De 11 a 30 & De $\$ 4,01$ a $\$ 100$ & 93 \\
\cline { 2 - 5 } & $\begin{array}{c}\text { Industria y } \\
\text { servicios }\end{array}$ & De 11 a 50 & De $\$ 100,01$ a $\$ 100$ & 95 \\
\hline \multirow{3}{*}{ Mediana $\$ 250$} & 235 \\
\cline { 2 - 5 } & Comercio & De 31 a 100 & De $\$ 100,01$ a $\$ 250$ & 235 \\
\cline { 2 - 5 } & Industria & De 51 a 100 & De \$100,01 a \$250 & 250 \\
\hline
\end{tabular}

Fuente: Secretaría de Economía de México (2009).

En 2016 el aporte de este sector al PIB nacional fue de $3 \%$, en tanto al PIB manufacturero fue de $18 \%$; un $20 \%$ de la inversión extranjera directa llegó a este sector y su participación en las exportaciones fue de 27\% (ProMéxico, 2015a, 2016). Con lo anterior se muestra que es un sector dinámico y en crecimiento en la economía mexicana.

Las principales empresas filiales de armadoras y de autopartes de Norteamérica, Europa y Asia se han instaurado en México, buscando garantizar entregas "justo a tiempo" y facilitando la producción flexible que requieren las armadoras. Entre las empresas establecidas destacan: BMW, Chrysler, Delphi, Denso, Eaton, Ford, General Motors, Honda, Magna, Mercedes Benz, Nissan, Robert Bosch, Toyota, Visteon, Volkswagen, entre otros.

\section{Las pymes de tecnologías de la información y la comunicación}

De acuerdo con datos de la Secretaría de Economía de México, las tecnologías de la información y las comunicaciones (TIC) son capaces de elevar el PIB hasta en $5 \%$, dado que es un sector en el que los cambios y la evolución no se detienen; prueba de esto es que el $90 \%$ de los dispositivos cuentan con sistemas integrados. Para 2020 se esperan 40.000 millones de chips con software embebido en el ámbito mundial. El mercado de videojuegos para 2013 alcanzó cerca de 73 billones de dólares.
En México existen 115 millones de dispositivos; sin embargo, no es un país desarrollador de contenidos y tecnología. Las redes sociales y los dispositivos móviles son los mercados de mayor crecimiento anual, con $10,6 \%$ y $13,8 \%$, respectivamente. El valor total de la industria de medios en México asciende a 54.000 millones de pesos, con un crecimiento anual de $8 \%$. Sin embargo, los indicadores básicos de penetración - banda ancha (10\%), telefonía móvil (82\%), telefonía fija (17\%) y computadoras (18\%) - son inferiores a países con las mismas condiciones de desarrollo económico (Palacios, Flores y García, 2013).

De acuerdo con ProMéxico (2015b), este sector en México tiene un valor de mercado de 20,7 billones de dólares, con un total de 3093 unidades económicas que generan 565.989 empleos directos; cuenta además con 639 centros de desarrollo certificados. Este sector es importante, pues las TIC han optimizado los procesos en todas las áreas y generado productividad; asimismo, han agilizado las comunicaciones, mejorado y brindado soporte al trabajo en equipo, y apoyado en gran manera las funciones estratégicas y operativas en las organizaciones.

\section{La administración del efectivo en las pymes}

El flujo de efectivo puede hacer que sobreviva o quiebre una empresa; por eso es un gran error no 
controlar el saldo de caja y los flujos de efectivo. Cuando el pequeño empresario se enfrenta a un faltante de efectivo, recurre a fondos propios, retrasa los pagos a proveedores o empleados, agiliza la cobranza de cartera, etc. Para evitar estos problemas, las pymes necesitarán establecer objetivos claros y concretos: no trabajar con una sola entidad financiera, adelantarse y prever necesidades, realizar operaciones transparentes, etc. (Saavedra, 2011; Opiela, 2006).

El flujo de efectivo está diseñado para mostrar qué tan bien la empresa está administrando su dinero; para ello, se calcula su liquidez y se les resta a los ingresos las salidas de dinero erogado por la empresa. Las pequeñas empresas deben realizar proyecciones de flujo de efectivo a tres años. Según Bangs (1978), la forma en que ello se debe elaborar es la siguiente:

a) Resumen de tres años pasados.

b) Proyección del primer año por mes. Si el negocio no alcanzó el punto de equilibrio en el primer año, se debe continuar haciendo la proyección mensual hasta que se llegue al punto de equilibrio.

c) Los años dos y tres se proyectan por cuartos (Vargas, 2007): las futuras necesidades de efectivo y las posibles fuentes en las que ha de ser obtenido.

Es necesario analizar cada concepto para determinar el importe de origen y la aplicación de efectivo en cada caso, en partidas como las siguientes (Vargas, 2007): efectivo en caja y bancos; cuentas por cobrar; deudores diversos; inventario de mercancía; inventario de materias primas; inventario de producción en proceso; inventario de artículos terminados; inmuebles, maquinaria y equipos; depreciación acumulada de inmuebles, maquinaria y equipos; proveedores; acreedores diversos; capital social; ingresos por venta; costo de venta; gastos de operación, y gastos financieros.

Un punto clave para las empresas es saber que a pesar del hecho de que existe una venta de buena fe, se ha realizado el envío de las mercancías, se registraron contablemente las ganancias, se emitieron las facturas, etc., no se ha afectado el flujo de efectivo de la empresa hasta tanto el cliente no haya pagado el importe de la venta realizada (Loscalzo, 1994).

\section{La liquidez y rentabilidad de la empresa y el ciclo de conversión de efectivo}

Lyroudi y McCarty (1993) encontraron que el ciclo de conversión de efectivo en las pymes está relacionado negativamente con la ratio de liquidez. Los resultados de sus estudios indican también que existen diferencias entre el ciclo de conversión de efectivo de empresas industriales, comerciales y servicios. En esto último coinciden con Konuk y Zeren (2014), que afirman que cada sector cuenta con un ciclo diferente de conversión de efectivo.

Muscettola (2014), a través de un estudio empírico, determinó que el ciclo de conversión de efectivo de las pymes manufactureras en Italia está relacionado con la rentabilidad de la empresa. Los resultados igualmente mostraron que el periodo promedio de cobranza se encuentra relacionado positivamente con la rentabilidad, con lo cual se viene abajo la idea de que hay que minimizar el ciclo de conversión de efectivo para aumentar la rentabilidad. Lo anterior sugiere que el crédito puede ser utilizado como una estrategia para mejorar la rentabilidad de las empresas. Konuk y Zeren (2014) también señalan que el ciclo de conversión de efectivo tiene impacto en la rentabilidad

Por su parte, Yasir, Majid y Yosuaf (2014) identificaron que el periodo de cobro a clientes se utiliza como indicador de eficiencia; es decir, se mide la capacidad de optimizar la gestión de cobranza de modo tal que se cuente con efectivo a la mayor brevedad posible. El hallazgo empírico de su estudio fue que el periodo de cobro a clientes tiene una relación negativa con el rendimiento de activos y el periodo de conversión de inventarios; esto es, se sugiere que la rentabilidad puede crecer si se reduce el tiempo concedido a los clientes y el tiempo del inventario (en otras palabras, se optimiza el proceso productivo). Tales conclusiones coinciden con lo encontrado por Kroes y Manikas (2014): 
las cuentas por cobrar (medidas en días de ventas) y las reducciones de inventario se relacionan positivamente con el desempeño de la empresa.

Ebben y Jonhson (2011) analizaron la relación entre el ciclo de conversión de efectivo, las inversiones de capital y la liquidez y desempeño de las pequeñas empresas durante un periodo de tres años, en empresas industriales y comerciales. Los resultados revelaron que el ciclo de conversión de efectivo es un factor significativo en el desempeño de las pequeñas empresas, en sintonía con lo concluido por Bolek, Kacprzyk y Wolski (2015); sin embargo, los propietarios de estas empresas no son proactivos en la administración del ciclo de efectivo. Esta investigación destaca la relevancia del ciclo de conversión de efectivo como herramienta de gestión proactiva de los pequeños propietarios de empresas.

Manoori y Muhammad (2012) confirmaron que las empresas con un ciclo de conversión de efectivo más largo son las que tienen mayor rentabilidad. Por el contrario, Ahmad y Raza (2012) establecieron una relación negativa entre el ciclo de conversión de efectivo y la rentabilidad medida en términos del rendimiento de activos totales. Por su parte, Dakros y Kallandranis (2007) encontraron que el impacto de los gastos de inversión en el flujo de efectivo se exacerba durante periodos de pesimismo, por lo que se puede inferir que las expectativas del entorno influyen en las decisiones de inversión.

Faris y Nassem (2013) presentan una investigación de empresas del sector industrial en Jordania, donde corroboraron la existencia de una importante relación directa entre el ciclo de conversión de efectivo y la deuda, el mercado, la productividad, la liquidez y los dividendos. Por último, Owolabi y Obida (2012) realizaron un estudio que muestra que la administración de la liquidez - medida en términos de políticas de crédito de la empresa, administración del flujo de efectivo y ciclo de conversión de efectivo- tiene un impacto importante en la rentabilidad; concluyen que los administradores pueden incrementar la rentabilidad aplicando buenas políticas de crédito, ciclos de conversión de efectivo cortos y procedimientos eficientes de administración del flujo de efectivo.

\section{El ciclo de conversión de efectivo}

Para explicar el ciclo de conversión de efectivo, es necesario partir del ciclo operativo (CO), entendido como el tiempo transcurrido entre el inicio del proceso productivo (con introducción de la materia prima y la mano de obra) y la recuperación del efectivo por la vía del cobro de las cuentas por cobrar generadas con la venta de los productos terminados (Saavedra, 2011). Se integra de la siguiente manera:

a) Días promedio de inventario (EPI).

b) Días promedio de cobro (PPC).

El CO se integra por la suma de los días promedio del inventario (DPI) y los días promedio de cobro (DPC):

$$
\mathrm{CO}=\mathrm{DPI}+\mathrm{DPC}
$$

Como se señaló, el CO de la empresa son los días entre el momento en que ingresa la materia prima al proceso productivo y el momento en que se cobra el efectivo. A continuación se explica en forma más detallada este término. Los conceptos centrales por utilizar son:

a) Tiempo de consumo: desde la incorporación de la materia prima hasta los productos en proceso.

b) Tiempo de producción: desde los productos en proceso hasta la obtención de productos terminados.

c) Tiempo de ventas: desde los productos terminados hasta la entrega a los clientes (cuentas por cobrar).

d) Tiempo de cobro: desde la entrega a clientes hasta el retorno de efectivo mediante la cobranza.

Por su parte, el ciclo de conversión del efectivo (CCE) son los días que tarda las empresas en recuperar el efectivo que invirtieron para generar el producto o servicio, considerando el $\mathrm{CO}$ y los días promedio de pagos (DPP). Este último es el plazo que requiere la empresa para cumplir con 
sus adeudos a proveedores de materias primas y otros a crédito. Lo recomendable es que la empresa pueda realizar todo el proceso de transformación, venta y cobranza en un periodo no mayor al periodo promedio de pagos; de otro modo deberá recurrir a recursos internos o externos para cumplir con sus obligaciones de corto plazo:

$$
\mathrm{CCE}=\mathrm{CO}-\mathrm{DPP}=\mathrm{DPI}+\mathrm{DPC}-\mathrm{DPP}
$$

Por ejemplo: si suponemos que los días promedio del inventario son 25 , los días promedio de cobro son 55 y los días promedio de pagos son 15, entonces se tiene:

$$
\mathrm{CCE}=25+55-15=65
$$

En la anterior fórmula, 65 días es el ciclo de conversión de efectivo. Como se puede ver, se redujo el tiempo respecto del ciclo operativo, debido a que se tiene un plazo para pagar a los proveedores, de forma tal que se obtiene un financiamiento espontáneo. Es necesario aclarar que cuanto más corto sea el ciclo de conversión de efectivo, menos recursos líquidos se necesitan para financiar las operaciones de la empresa.

\section{METODOLOGÍA}

Para el desarrollo de esta investigación se utilizó el método de caso múltiple, mediante la recolección y el análisis de datos de 17 empresas (9 del sector automotor y 8 del sector de $\mathrm{TI}$ ), con el fin de proponer un modelo de flujo de efectivo de acuerdo con sus características y necesidades específicas. En este artículo, por cuestión de espacio, se presentan en detalle solo dos empresas; sin embargo, en la tabla 4 se muestra el efecto de la aplicación para todas las empresas. De acuerdo con Yin (2003), este diseño tiene sus ventajas y desventajas: en general, la evidencia creada a partir de este tipo de estudios se considera robusta y fiable, pero también puede llevar mucho tiempo y costos su realización. Adicionalmente, como ventaja, se puede efectuar el estudio a profundidad en cada empresa y de este modo establecer sus requerimientos particulares.

\section{MODELO PROPUESTO DE FLUJO DE EFECTIVO}

En muchas ocasiones, el empresario está muy embebido en la operación de la empresa y no hace reflexiones sobre el dinero que tiene; pocas veces los empresarios hacen proyecciones para analizar la situación futura de su negocio. Esta herramienta puede ser usada para vincularlos con algún inversionista o banco, una vez se tenga la certeza de si la empresa puede acceder a un préstamo bancario o a un requerimiento financiero. Asimismo, es un instrumento valioso para el consultor que puede ayudar a las empresas al "buen morir", es decir, cuando las empresas ven que en sus proyecciones solo hay números rojos y no existe una estrategia clara de cómo evitar tal situación; entonces, el mismo empresario se da cuenta de que no es conveniente seguir operando de la forma en que lo está haciendo hasta el momento.

Después de las interacciones con los empresarios y el análisis de sus necesidades, se buscó desarrollar la metodología enfocada a pymes mexicanas de los sectores automotor y $\mathrm{TI}$, como se muestra a continuación.

\section{Selección de rubros necesarios para la elaboración de la metodología de flujo de efectivo para pymes}

Un punto fundamental para el desarrollo de la metodología fue entender cuáles son los rubros de mayor importancia para empresas mexicanas de los sectores seleccionados e identificar cuáles de ellos serían claves para un análisis de mayor valor para las empresas. Una vez conocidas las necesidades de las empresas, y con base en la bibliografía consultada, se decidió dividir el flujo de efectivo como se muestra en la tabla 2. 
Tabla 2.

Esquema de flujo de efectivo

\begin{tabular}{|c|c|}
\hline Rubros & Ítems \\
\hline Ingresos & $\begin{array}{ll}\text { - } & \text { Efectivo disponible (inicio del mes) } \\
\text { - } & \text { Ventas en efectivo cliente } 1 \\
\text { - } & \text { Cuentas por cobrar cliente } 1 \\
\text { - } & \text { Cuentas en por cobrivo cliente } 2 \\
\text { - } & \text { Ventas en efectivo cliente } 2 \\
\text { - } & \text { Cuentas por cobrar cliente } 3 \\
\text { - } & \text { Otros clientes } \\
\text { - } \quad \text { Préstamos } \\
\text { - } \quad \text { Otra entradada de efectivo } \\
\end{array}$ \\
\hline Egresos & 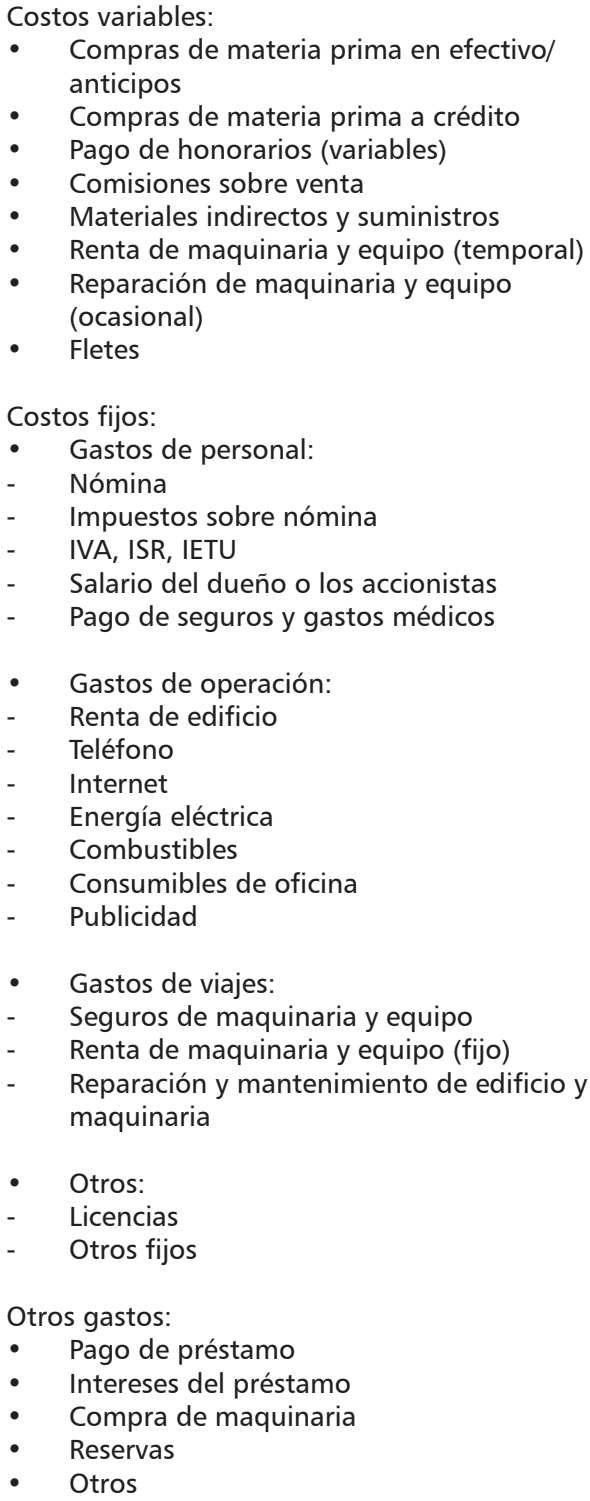 \\
\hline
\end{tabular}

Fuente: elaboración de los autores

\section{Elaboración de formato en Excel con fórmulas para la determinación automática de los valores}

Una vez se tuvieron los rubros determinados, se llevaron a una hoja en Excel con la finalidad de dar estructura al flujo de efectivo. En este punto fue indispensable buscar que la imagen de la herramienta fuese amigable, ya que Excel es usualmente relacionado con complejidad y normalmente los empresarios dejan de tener interés cuando se les obliga a poner información allí. Igualmente, una parte fundamental de la herramienta es lograr que sea clara y sencilla para los empresarios. Para ello se hizo un vaciado de todos los conceptos necesarios para el análisis, se estructuraron por grupos y luego se les dio un sentido descendente; es decir, se empieza con los clientes y las ventas, y después todas las entradas de efectivo; posteriormente se ponen todas las salidas de dinero, empezando con los gastos variables, y por último se encuentran los fijos; al final se pone un renglón que señale el flujo de efectivo del mes.

Tan pronto se tuvo una primera versión, la herramienta fue aplicada a un grupo de empresas que forman parte de los programas de la Fundación México-Estados Unidos para la Ciencia (FUMEC), con miras a obtener información financiera detallada y generar recomendaciones puntuales. Para esto se firmó un acuerdo de confidencialidad con las empresas que posibilitara acceder a sus datos financieros.

\section{Esquema metodológico de la generación del modelo}

Uno de los sectores más importantes del país es el automotor; de hecho, México es el octavo productor del mundo, contribuyendo al PIB en 4\%. Por otra parte, uno de los sectores con mayor crecimiento es el de las TIC, que incluso eleva el PIB mexicano hasta un 5\%. De ahí la importancia de la generación y aplicación de ese modelo para empresas de ambos sectores. Estas pymes en su mayoría son dirigidas por ingenieros o emprendedores que tienen muy 
poco conocimiento sobre contabilidad o cuestiones financieras, por lo que afrontan situaciones que se vuelven críticas:

- Pago de la nómina.

- Uso de tarjetas de crédito para cumplir con sus obligaciones.

- Liquidez para emprender nuevos proyectos.

- Contar con una estrategia de crecimiento bien definida.

Para una mejor comprensión, a continuación se presenta el esquema metodológico para el desarrollo del modelo de flujo de efectivo propuesto:

a) Diagnóstico de las operaciones financieras. Para ello, se tienen en cuenta los siguientes ítems:

- No lleva control de entradas y salidas, o lo hace de manera informal.

- No tiene al día las cuentas por cobrar a clientes.

- Lleva control de ingresos y egresos separadamente.

- Carece de relación la información del mes anterior con la del mes actual.

- La información del flujo de efectivo la manejan varias personas, y el director general no solicita información.

b) Reunión con el empresario. El objetivo de la reunión es conocer y aplicar una herramienta estratégica que pretende hacer notar la importancia del uso del flujo de efectivo para la empresa, tomando en cuenta su pasado, su presente y, sobre todo, su futuro. No pretende obtener el número exacto en la cuenta de cheques, sino tener una muy buena aproximación y que el empresario entienda cómo usar la herramienta; en suma, se busca concientizar y generar una cultura financiera en la Dirección e identificar situaciones críticas y problemas de ciclicidad. c) Instrumento. Se desarrolla un instrumento en Excel que contempla el análisis de ingresos, egresos y efectivo al final del cada mes.

d) Interacciones. Se inicia con preguntas puntuales, haciendo análisis de ingresos y egresos de la empresa. Respecto del análisis de los ingresos de efectivo, se tienen en cuenta los siguientes aspectos:
- Clientes principales y entradas de efectivo. No es lo mismo que facture $\$ 100.000$ en " $x$ " mes a que cobre esa cantidad en el mismo mes.

- Cuentas por cobrar.

- Engloba los demás clientes.

- Otras entradas de dinero.

- Se validan los ingresos de conformidad con el director.

- La empresa podrá agregar u omitir conceptos de acuerdo con las necesidades.

Por su parte, respecto al análisis de los egresos de efectivo, se consideran:

- Costos variables: materia prima, materiales indirectos, pago de honorarios variables (freelancers), renta de maquinaria, reparación de maquinaria y equipo ocasional, y fletes.

- Costos fijos: gastos de personal, egresos de nómina, impuestos sobre nómina, salario del dueño o los accionistas, pago de seguros y gastos médicos, pago de contador.

- Compara el crédito de los proveedores con el de sus clientes.

- Gastos de operación: renta del edificio, teléfono, internet, energía eléctrica, combustibles, consumibles de oficina, publicidad, gastos de viaje, seguros en maquinaria y equipo, renta de maquinaria y equipo de forma habitual (fija), y reparación y mantenimiento de edificio y maquinaria.

- Otros: licencias.

- Otros gastos: préstamos, intereses generados por préstamos, compra de maquinaria, reservas y algún otro gasto.

- Se validan los egresos de conformidad con el director/empresario.

e) Análisis financiero y recomendaciones. Se tienen en cuenta aspectos como los siguientes:

- Examina los porcentajes que representa cada rubro.

- Revisa el punto de equilibrio y la contribución marginal. 
- Compara los resultados con empresas del mismo sector.

- Comparte su experiencia y hace recomendaciones para la empresa.

f) Genera escenarios. Se consideran los siguientes aspectos:

- Algún nuevo proyecto (incluyendo la inversión y las ventas estimadas).

- Inversión de maquinaria y equipo.

- Aumento de personal.

- Automáticamente actualiza el efectivo disponible mensual, lo que permite visualizar los efectos de las decisiones.

- Entrega de reporte.

El análisis de flujo de efectivo se realiza con el objetivo de conocer la capacidad real de inversión de las empresas y de este modo soportar las actividades por realizar en los ámbitos nacional e internacional y en innovación. Se concluye con una propuesta de valor afinada para cada empresa. En la figura 1 se presenta esta metodología de modo esquemático para facilitar su comprensión.

\section{APLICACIÓN DE LA HERRAMIENTA}

\section{Caso 1: sector automotor, Nuevo León}

Se trata de una empresa del sector industrial dedicada a la fabricación de partes automotrices. Cuenta con 12 empleados. Los hallazgos en esta empresa fueron:

- Cliente 1: en todas las cotizaciones tienen que aplicar un porcentaje $4 \%$ abajo.

- Con Nacional Financiera (NAFIN), banca de fomento: el factoraje les puede costar hasta $12 \%$ (es un costo financiero).

- Cliente 2: facturan sin IVA, es parte de un grupo y ahora van a entrar con otro cliente más grande; con ellos son $40 \%$ de anticipo, $40 \%$ al final y $20 \%$ al liberar el producto.

- Crédito: les intenta cobrar de contado, sino una semana, luego quince días, luego un mes. Tiene dos facturas por cobrar

Figura 1.

Esquema metodológico de la generación del modelo de flujo de efectivo

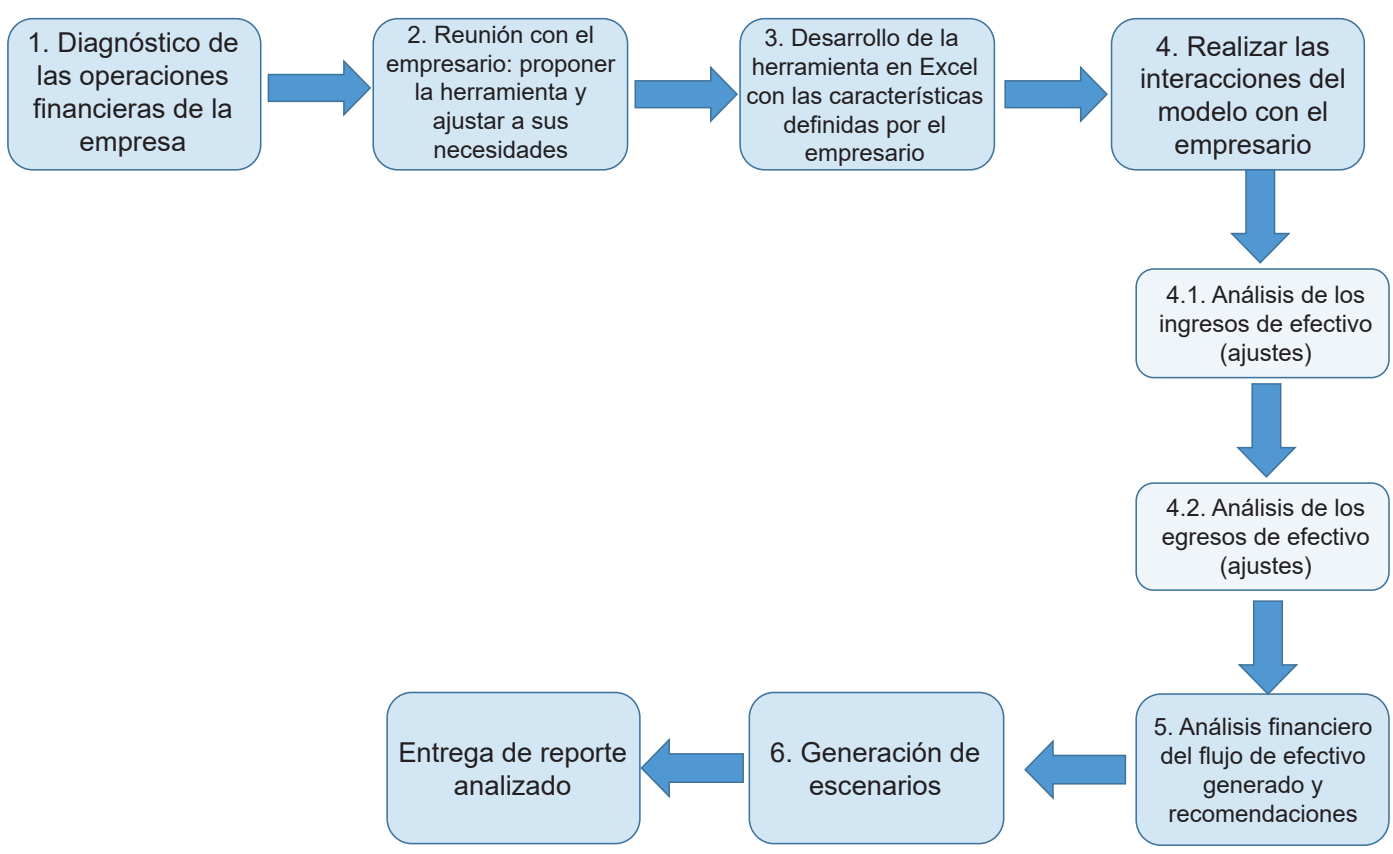

Fuente: elaboración de los autores. 
(febrero y mayo), que suman un total de 50.000 pesos.

- Préstamos: recurren a préstamos familiares; llevan $\$ 250.000$ en cuatro años.

Nota: es importante mencionar que el flujo que se hizo es el ideal porque se toma en enero: las facturas correspondientes a ese mes que se cobraron o están por cobrar como entrada, lo cual suma el total de lo facturado, menos el factoraje (en caso de ocurrir); se incluyen también los montos que efectivamente entraron en el mes (aunque hayan sido facturas de meses anteriores, o los anticipos que entraron en ese mes).

A la empresa se le hizo una serie de recomendaciones:

- El siguiente paso es que la empresa vacíe la información real y detallada del negocio en el flujo de efectivo para su análisis. La idea es que este ejercicio se realice de manera periódica como parte de la planeación y el control de la empresa, de manera tal que el comportamiento y las nuevas iniciativas del negocio se puedan trasladar en entradas y salidas de dinero, y de esta manera ver cómo se va a comportar "el medidor de gasolina".

- La recomendación es que el flujo de efectivo se extienda a un horizonte de 1 año con detalle mensual, y los siguientes 1-2 años por cuatrimestres. La utilidad de la herramienta estriba en que se actualice y utilice de manera cotidiana, de forma tal que las decisiones de negocio que se tomen estén basadas en cifras reales y que, a partir del análisis de desviaciones entre lo proyectado inicialmente y lo real, se puedan identificar áreas de oportunidad y mejora.

- Se debe recordar al empresario que la herramienta debe reflejar exactamente cómo entra el dinero, no lo facturado; en enero poner lo que se cobró en enero y tener en cuentas por cobrar lo que queda pendiente. Actualmente se tiene un acumulado grande de cuentas por cobrar, por lo que se sugiere que se ponga de enero a julio todo lo que realmente le entró a la empresa por cliente, y que se pongan todas las cuentas por cobrar al igual por cliente en el mes que se estime que se puedan cobrar. Esto tiene por finalidad generar objetivos de cobro e indicadores.

- Es gravísimo que no tengan un control de gastos, todo lo lleva el contador. Se sugiere llevar el control de aquí en adelante. Se le comentó al empresario la importancia de tener un control interno de sus gastos, dado que el crecimiento de la empresa será importante en los próximos meses; no tener un control de sus gastos puede llevar a que no se tenga flujo de efectivo para cubrir con sus obligaciones; sin saber las causas, no se podrá hacer eficiencias en gastos ni indicadores de medición.

- El dueño no tiene un sueldo. En el manejo del efectivo no es claro si mezclan dinero del empresario con el de la empresa (usan préstamos familiares). El dueño tiene un salario simbólico. El empresario debe evaluar su flujo de efectivo y establecer un salario. Es muy importante evitar que se mezcle el dinero del empresario con el de la empresa.

- Se detectaron cuentas por cobrar por $\$ 61.350$ dólares aproximadamente (tienen facturas desde enero). Es necesario tener un control específico de las cuentas por cobrar, estableciendo procesos de seguimiento e identificando los problemas por lo que no se están cobrando.

- Es muy importante que se pida al contador un desglose de gastos históricos de la empresa, y que se lleve un seguimiento tanto dentro de la empresa como con el contador; es decir, la herramienta de flujo de efectivo puede ser usada tanto por el contador como por la empresa, pero se debe tener un control de todos los ingresos y egresos. 
- La herramienta de flujo de efectivo puede apoyar a la empresa a tomar las decisiones en cuanto a compra de maquinaria y equipo; si se lleva a precisión la herramienta, se puede saber con cuánto dinero se cuenta y con cuánto dinero se contará, lo cual es factor para tomar decisiones.

- La compra de maquinaria, de acuerdo con el flujo que se obtuvo (recordar que no es flujo real), representa el $49,7 \%$ de las ventas, lo cual muestra un alto apalancamiento de la empresa (tiene muchas deudas). Es indispensable que se tome en cuenta esto, ya que si se siguen adquiriendo activos fijos sin cobrar lo que se ha vendido, llegará un punto en el que la pyme tenga que pedir demasiados préstamos que disminuirán considerablemente su utilidad.

- En caso de presentarse un excedente al final del año, se pueden ir explorando alternativas para su uso: iniciar un fondo de I\&D que sirva para desarrollar nuevos productos o servicios, o tener un fondo de reserva para hacer frente a posibles contratiempos de flujo. También se puede explorar la posibilidad de invertir los excedentes de flujo en instrumentos financieros de corto plazo, considerando rendimientos e impuestos.

- Se recomienda desglosar los ingresos por cliente para poder hacer un mejor análisis al respecto; con la información recogida podría hacerse una mejor proyección para los próximos meses y diseñar una estrategia de comercialización por cliente.

- Se recomienda elaborar el flujo de efectivo del año pasado para hacer un comparativo y ver si va a haber un crecimiento en este año; habría también que analizar la relación de ese crecimiento contra los márgenes de utilidad.

En la tabla 3 se condensa el resultado del flujo de la empresa (solo se muestran tres meses, por motivo de espacio).

Tabla 3.

Análisis de flujo de efectivo en sector automotor, Nuevo León

\begin{tabular}{|c|c|c|c|}
\hline Ingresos & Enero & Febrero & Marzo \\
\hline Efectivo disponible (inicio del mes) & 0 & 67.459 & 82.437 \\
\hline Ventas en efectivo Bosch & 240.536 & 113.906 & 164.416 \\
\hline Cuentas por cobrar Bosch & 0 & 0 & 0 \\
\hline Bosch Toluca & & 54.492 & \\
\hline \multicolumn{4}{|l|}{ Cuentas por cobrar Bosch Toluca } \\
\hline Ventas en efectivo ATS & 33.670 & & \\
\hline Cuentas por cobrar ATS & 0 & 0 & 0 \\
\hline Ventas en efectivo FILPE & & 2.645 & \\
\hline \multicolumn{4}{|l|}{ Cuentas por cobrar FILPE } \\
\hline DEMAC & & & 8294 \\
\hline \multicolumn{4}{|l|}{ Cuentas por cobrar DEMAC } \\
\hline Denso & & & 8355 \\
\hline \multicolumn{4}{|l|}{ Cuentas por cobrar Denso } \\
\hline Otros clientes & 0 & 0 & 0 \\
\hline Préstamos & 7000 & & 51.600 \\
\hline Aportación del dueño & 0 & 0 & 0 \\
\hline \multicolumn{4}{|l|}{ Bonificación del banco } \\
\hline Otra entrada de efectivo & 0 & 0 & 17.139 \\
\hline Total de efectivo recibido & 281.206 & 171.043 & 249.804 \\
\hline Total de efectivo disponible & 281.206 & 238.502 & 332.241 \\
\hline
\end{tabular}




\begin{tabular}{|c|c|c|c|}
\hline Egresos & Enero & Febrero & Marzo \\
\hline Maquinados & 113.816 & 41.157 & 19.966 \\
\hline \multicolumn{4}{|l|}{ Otros gastos de maquinados } \\
\hline Materia prima & 18.345 & 10.177 & 15.719 \\
\hline Partes comerciales & 3555 & 6445 & 0 \\
\hline Tratamientos térmicos & 1452 & 2260 & 0 \\
\hline Acabados & 495 & 1035 & 230 \\
\hline Fletes & 0 & 0 & 0 \\
\hline Herramientas & 5474 & 5254 & 3329 \\
\hline Empaques & 1540 & 445 & 1086 \\
\hline Ferretería & 2000 & 3205 & 1000 \\
\hline Vendedor por comisión & & & 700 \\
\hline Otros costos & 1110 & 1887 & 0 \\
\hline Costos variables & 147.787 & 71.865 & 42.030 \\
\hline Gastos de personal & 32.400 & 40.060 & 43.039 \\
\hline Nómina & 32.400 & 21.609 & 37.459 \\
\hline Seguridad Social (IMSS) & 0 & 2093 & 0 \\
\hline \multicolumn{4}{|l|}{ Impuesto $2 \%$ sobre nómina } \\
\hline Impuestos: IVA ISR IETU & & 5358 & \\
\hline Contador & & 5000 & \\
\hline Notario & & 6000 & \\
\hline Préstamos a personal & & & 2000 \\
\hline \multicolumn{4}{|l|}{ Horas extras } \\
\hline Gastos médicos mayores & & 0 & 3580 \\
\hline Gastos de operación & 33.232 & 43.812 & 18.852 \\
\hline Renta de edificio & 12.000 & 12.000 & 12.000 \\
\hline \multicolumn{4}{|l|}{ Alarma } \\
\hline Teléfono TELMEX & 0 & 0 & 0 \\
\hline TELUM & 2103 & 5452 & 1978 \\
\hline Internet & 0 & 0 & 0 \\
\hline Electricidad CFE & 0 & 3142 & 0 \\
\hline Agua & 0 & 389 & 0 \\
\hline Gasolina & 7000 & 2350 & 1000 \\
\hline Consumibles de oficina & 2918 & 5370 & 2000 \\
\hline Equipo de oficina & & 2205 & 0 \\
\hline Viáticos & 8000 & 9469 & 0 \\
\hline \multicolumn{4}{|l|}{ Mantenimiento de camioneta y multas } \\
\hline Construcción de oficinas & & 0 & \\
\hline Publicidad & 1145 & 3000 & 1874 \\
\hline Paquetería y aduana & 66 & 435 & 0 \\
\hline Otros & 0 & 0 & 0 \\
\hline Licencias & 0 & 0 & 0 \\
\hline Costos fijos & 65.632 & 83.872 & 61.891 \\
\hline Total gastos de operación & 213.419 & 155.737 & 103.921 \\
\hline Otros gastos & 328 & 328 & 328 \\
\hline Pago de préstamo & 0 & 0 & 0 \\
\hline Intereses del préstamo & 0 & 0 & 0 \\
\hline Comisiones bancarias & 328 & 328 & 328 \\
\hline \multicolumn{4}{|l|}{ Comisiones de cheques } \\
\hline Reservas & 0 & 0 & 0 \\
\hline \multicolumn{4}{|l|}{ Maquinaria y equipo } \\
\hline Otros & 0 & 0 & 0 \\
\hline Total de efectivo pagado & 213.747 & 156.065 & 104.249 \\
\hline Efectivo al final del mes & 67.459 & 82.437 & 227.992 \\
\hline
\end{tabular}

Fuente: elaboración de los autores. 


\section{Caso 2: tecnologías de la información, Nuevo León}

Esta empresa ofrece servicios TIC a las pymes. Los hallazgos que se encontraron fueron los siguientes:

- Cliente 1: es un organismo intermedio"; la empresa vendió en punto de venta a 100 tienditas. Tiene un problema con retraso de pago, dado que el cliente final no ha cancelado lo que adeuda (los abarroteros). Esto representa el $75 \%$ de los ingresos de la empresa.

- Cliente 2: tiene un gran proyecto por el que cobrarán 1700 dólares mensuales.

- Cliente 3: tiene un aliado estratégico para el desarrollo de un proyecto, con lo cual va a crecer hasta llegar a 30.000.

- Proveedores: tiene proveedores que pagan de contado; otros lo hacen a 15, 20 y 30 días.

- Tiene pagos fijos, lo que le permite contar con ingresos fijos; es el caso del cliente 3 y el pago tras entrega en el caso del cliente 2.

- Generalmente cobran anticipos del $50 \%$, lo otro lo prorratean; la idea es que termine el desarrollo cuando se finaliza el cobro.

- Tiene una estrategia diferente a las empresas antes analizadas: trata de "jugar" con el dinero del cliente, porque trata de cobrar a menos días que los que paga.

A la empresa se le hicieron una serie de recomendaciones:

- Se hizo un flujo de efectivo forzado en un principio, muy interesante ya en su desarrollo; sin embargo, es importante recordar que dentro del flujo existen saldos en rojo en meses pasados, lo cual no corresponde a la realidad (de algún lado tuvo que salir dinero para cubrir sus deudas). Es importante señalar que si el

1 Son entes autorizados por el Gobierno Federal para brindar asesoría a las pymes; pueden ser entes públicos o privados de los sectores financiero, educativo, empresarial, de gobierno, entre otros. empresario quiere que la herramienta le funcione adecuadamente, debe usar los números reales.

- Se debe tener cuidado porque su mayor cliente es el que peor paga. Una de las líneas posibles de acción es la búsqueda de clientes o el establecimiento de políticas de cobro más estrictas.

- Al empresario se le hizo muy interesante el tema de los porcentajes. En el ejercicio realizado se tuvo un costo de personal del $12,88 \%$, aunque el empresario comentó que debería estar en $5 \%$. Si lo que se busca es un control exhaustivo de los gastos el flujo, debe tener los datos reales para que sea funcional en la proyección de sus egresos.

- Es una empresa que a finales de año será extremadamente rentable; se prevén muchas entradas de dinero; no se proyectaron los gastos adecuadamente, pero tal como se vio, pasaría de cobrar \$300.000 mensuales a \$1.200.000 mensuales ( $400 \%$ más). Ante esto, se deben establecer indicadores u objetivos para que esto se dé, y estimar los gastos para observar el escenario de cuánto dinero se va a necesitar para cubrir gastos mínimos. Tener en cuenta esta recomendación es fundamental, porque la pyme está teniendo problemas de cobranza, y si uno de estos proyectos no se cobra tal como se estima, puede provocar que la empresa no tenga el flujo para cubrir esos gastos y deba recurrir a préstamos.

- Es igualmente importante establecer correctamente cuándo se estarán realizando las inversiones que se estimaron en la reunión. Estas inversiones fuertes pueden ser marginales siempre y cuando se cobre lo que se vende.

El flujo de la empresa quedó como se muestra en la tabla 4 (solo se presentan tres meses, por motivos de espacio). 
Tabla 4.

Análisis de flujo de efectivo en pyme de tecnologías de la información, Nuevo León

\begin{tabular}{|c|c|c|c|c|c|c|}
\hline \multirow{2}{*}{$\begin{array}{c}\text { Ingresos } \\
\text { Efectivo disponible (inicio del mes) }\end{array}$} & \multicolumn{2}{|r|}{ Enero } & \multicolumn{2}{|r|}{ Febrero } & \multicolumn{2}{|r|}{ Marzo } \\
\hline & $\$$ & 0 & $\$$ & 1.674 .260 & $\$$ & 1.411 .820 \\
\hline Ventas en efectivo San Nicolás (11\%) & $\$$ & - & $\$$ & - & $\$$ & 174.570 \\
\hline Cuentas por cobrar San Nicolás & $\$$ & - & $\$$ & - & $\$$ & - \\
\hline Ventas en efectivo Bustamante (10\%) & $\$$ & 158.700 & & & & \\
\hline Cuentas por cobrar Bustamante & $\$$ & - & $\$$ & - & $\$$ & - \\
\hline Ventas en efectivo Canacope (75\%) & $\$$ & 1.587 .000 & $\$$ & 200.000 & $\$$ & 200.000 \\
\hline \multicolumn{7}{|l|}{ Cuentas por cobrar Canacope } \\
\hline \multicolumn{7}{|l|}{ Nielsen } \\
\hline \multicolumn{7}{|l|}{ Mi Tiendita } \\
\hline \multicolumn{7}{|l|}{$\mathrm{HC}$} \\
\hline Otros clientes & & & & & $\$$ & - \\
\hline Préstamos & $\$$ & 400.000 & & & $\$$ & - \\
\hline Aportación del dueño & $\$$ & - & $\$$ & - & $\$$ & - \\
\hline Otra entada de efectivo & & & $\$$ & - & $\$$ & - \\
\hline Total de efectivo recibido & $\$$ & 2.145 .700 & $\$$ & 200.000 & $\$$ & 374.570 \\
\hline Total de efectivo disponible & $\$$ & 2.145 .700 & $\$$ & 1.874 .260 & $\$$ & 1.786 .390 \\
\hline \multicolumn{7}{|l|}{ EGRESOS } \\
\hline Compra de equipo hardware & $\$$ & 150.000 & $\$$ & 150.000 & $\$$ & 960.135 \\
\hline Compras materia prima a crédito & $\$$ & - & $\$$ & - & $\$$ & - \\
\hline Materiales Indirectos y suministros & $\$$ & - & $\$$ & - & $\$$ & - \\
\hline Pago de honorarios (variables) & $\$$ & - & $\$$ & - & $\$$ & - \\
\hline Renta de maquinaria y equipo (temporal) & $\$$ & - & $\$$ & - & $\$$ & - \\
\hline \multicolumn{7}{|l|}{ Reparación de maquinaria y equipo (ocasional) } \\
\hline \multicolumn{7}{|l|}{ Fletes } \\
\hline Costos variables & $\$$ & 150.000 & $\$$ & 150.000 & $\$$ & 960.135 \\
\hline Gastos de personal & $\$$ & 275.000 & $\$$ & 275.000 & $\$$ & 275.000 \\
\hline Nómina & $\$$ & 200.000 & $\$$ & 200.000 & $\$$ & 200.000 \\
\hline Impuestos sobre nomina & $\$$ & 50.000 & $\$$ & 50.000 & $\$$ & 50.000 \\
\hline IETU ISR IVA & $\$$ & 15.000 & $\$$ & 15.000 & $\$$ & 15.000 \\
\hline Contador & $\$$ & 10.000 & $\$$ & 10.000 & $\$$ & 10.000 \\
\hline \multicolumn{7}{|l|}{ Salario del dueño o accionistas } \\
\hline Pago de seguros y gastos médicos & & & $\$$ & - & $\$$ & - \\
\hline Gastos de operación & $\$$ & 46.440 & $\$$ & 37.440 & $\$$ & 46.440 \\
\hline Renta de edificio & $\$$ & 9890 & $\$$ & 9890 & $\$$ & 9890 \\
\hline Teléfono internet y Axtel & $\$$ & 6000 & $\$$ & 6000 & $\$$ & 6000 \\
\hline Energía eléctrica & $\$$ & 9000 & & & $\$$ & 9000 \\
\hline Combustibles & $\$$ & 5000 & $\$$ & 5000 & $\$$ & 5000 \\
\hline Consumibles de oficina & $\$$ & 3000 & $\$$ & 3000 & $\$$ & 3000 \\
\hline Publicidad & $\$$ & 1000 & $\$$ & 1000 & $\$$ & 1000 \\
\hline Gastos de viajes & $\$$ & 10.000 & $\$$ & 10.000 & $\$$ & 10.000 \\
\hline Seguros en maquinaria y equipo & $\$$ & 550 & $\$$ & 550 & $\$$ & 550 \\
\hline Renta de maquinaria y equipo (fijo) & & & $\$$ & - & & \\
\hline Mantenimiento de autos & $\$$ & 2000 & $\$$ & 2000 & $\$$ & 2000 \\
\hline Otros & $\$$ & - & $\$$ & - & $\$$ & - \\
\hline Licencias & $\$$ & - & $\$$ & - & $\$$ & - \\
\hline \multicolumn{7}{|l|}{ Otros } \\
\hline Costos fijos & $\$$ & 321.440 & $\$$ & 312.440 & $\$$ & 321.440 \\
\hline Total gastos de operación & $\$$ & 471.440 & $\$$ & 462.440 & $\$$ & 1.281 .575 \\
\hline Otros gastos & $\$$ & - & $\$$ & - & $\$$ & - \\
\hline
\end{tabular}




\begin{tabular}{|c|c|c|c|c|c|c|}
\hline Ingresos & & Enero & & Febrero & & Marzo \\
\hline Pago de préstamo & $\$$ & - & $\$$ & - & $\$$ & - \\
\hline Intereses del préstamo & $\$$ & - & $\$$ & - & $\$$ & - \\
\hline Compra de maquinaria & $\$$ & - & $\$$ & - & $\$$ & - \\
\hline Hosting & & & & & & \\
\hline Reservas & $\$$ & - & $\$$ & - & $\$$ & - \\
\hline Otros & $\$$ & - & $\$$ & - & $\$$ & - \\
\hline Total de efectivo pagado & $\$$ & 471.440 & $\$$ & 462.440 & $\$$ & 1.281 .575 \\
\hline Efectivo al final del mes & $\$$ & 1.674 .260 & $\$$ & 1.411 .820 & $\$$ & 504.815 \\
\hline
\end{tabular}

Fuente: elaboración de los autores.

\section{Componentes de la herramienta}

\section{Ingresos}

Todo tipo de entrada de dinero real percibido por la empresa en los diferentes meses del año:

a) Efectivo disponible al inicio del mes: dinero con el que la empresa iniciará sus actividades del mes. Esta cantidad es la diferencia de ingresos y egresos del mes anterior.

b) Ventas en efectivo cliente 1: se refiere a lo que le ingresa de dinero a la empresa; es importante diferenciar entre lo que se factura y lo que realmente entra a la empresa; en este caso, se registra la entrada real de dinero.

c) Cuentas por cobrar cliente 1: se anotan todos los ingresos de dinero que quedan pendientes por cobrar por parte de la empresa.

d) Ventas en efectivo cliente 2 y cliente 3 : son ingresos de diferentes clientes de la empresa.

e) Otros clientes: en este rubro se deben poner todos aquellos ingresos de los clientes que no fueron contemplados en los rubros anteriores.

f) Préstamos: son los ingresos percibidos por la empresa vía préstamos bancarios.

g) Aportaciones del dueño: ingresos que percibe la empresa por concepto de la inyección de efectivo vía dueños o accionistas. h) Otra entrada de efectivo: son los ingresos que entran a la empresa por cualquier otro concepto que no ha sido tomado en cuenta.

Es importante señalar que estos rubros son solo ejemplos por utilizar durante el desarrollo del ejercicio; el empresario podrá utilizar cualquier otro concepto que considere conveniente durante las dos horas en las que dura el ejercicio. Esta metodología no pretende obtener el número exacto que tiene la empresa en su cuenta de cheques; el objetivo es tener una buena aproximación a la realidad y que el empresario entienda cómo usar la herramienta.

\section{Egresos}

Son todo tipo de salidas de dinero que tenga la empresa durante un año; se encuentran divididos en tres rubros:

a) Costos variables: todos los egresos de la empresa presentados cada mes dependiendo de las circunstancias; es decir, no se presentan de manera común en cada uno de los meses. Dentro de los costos variables se encuentran los siguientes rubros:

- Compras de materia prima en efectivo: salida de efectivo por parte de la empresa por concepto de adquisiciones de materia prima para la elaboración de su producto final, pagada de contado. 
- Compras de materia prima a crédito: egresos de la empresa para la adquisición de materia prima pero que no se efectúan de contado, sino que se adquieren vía crédito; se ponen en el mes donde se pretende realizar el pago de dichas compras.

- Materiales indirectos y suministros: egresos por concepto de suministros o materiales que no están directamente relacionados con el producto final.

- Pago de honorarios variables: pago a personas que no forman parte de la nómina y que son contratadas solo en ocasiones específicas para el desarrollo de proyectos específicos.

- Renta de maquinaria y equipo temporal: egresos como consecuencia de renta de equipo que solo ocurre en ocasiones específicas.

- Reparación de maquinaria y equipo: salidas de dinero efectuadas por la empresa por concepto de reparaciones de su equipo y maquinaria que no es contemplada como periódica, sino que se presenta ocasionalmente.

- Fletes: egresos mensuales de la empresa efectuados por concepto de fletes que van en función de los productos que vende la empresa.

b) Costos fijos: todos los egresos de la empresa ocurridos irremediablemente mes a mes, resultado de la operación:

- Gastos de personal:

- Nómina: egresos que realiza la empresa mensualmente por concepto de pago a sus trabajadores.

- Impuestos sobre nómina: salidas de dinero como consecuencia de los impuestos relacionados con la nómina.

- Salario del dueño o los accionistas: egresos de la empresa emitidos por concepto del pago del salario del dueño o de los accionistas de la institución.
- Pago de seguros y gastos médicos: dinero que la empresa gasta por concepto de seguros de todo tipo o gastos médicos.

- Gastos de operación: egresos relacionados con la operación de la empresa. En este rubro entran conceptos como renta, teléfono, internet, energía eléctrica, combustibles, consumibles de oficina, publicidad, gastos de viaje, seguros en maquinaria y equipo, renta de maquinaria y equipo, reparación y mantenimiento de edificios y maquinaria. Al igual que en las demás secciones, se pueden agregar celdas con base en la necesidad de cada una de las empresas, ya que puede pasar que los rubros no sean usados por la empresa. Por lo tanto, se anotarán los conceptos usados por la empresa.

- Otros costos fijos: en este campo se concentran los gastos de la empresa no mencionados con anterioridad y que son recurrentes cada mes.

\section{Otros gastos}

Son todos los egresos de la empresa que no tienen una relación directa con su operación. Se separa de todos los demás gastos justamente para denotar la diferencia entre los gastos de operación y los gastos que no lo son. Entre estos gastos se sugieren: pago de préstamos (ya sean bancarios o de otra índole), intereses del préstamo, compra de maquinaria, reservas y otras.

\section{RESULTADOS DE APLICACIÓN DEL MODELO PROPUESTO}

Se aplicó la herramienta a 17 empresas y se identificaron impactos en cada una de ellas; con esto se realizó una matriz (tabla 5) que señala el beneficio obtenido por empresa. 


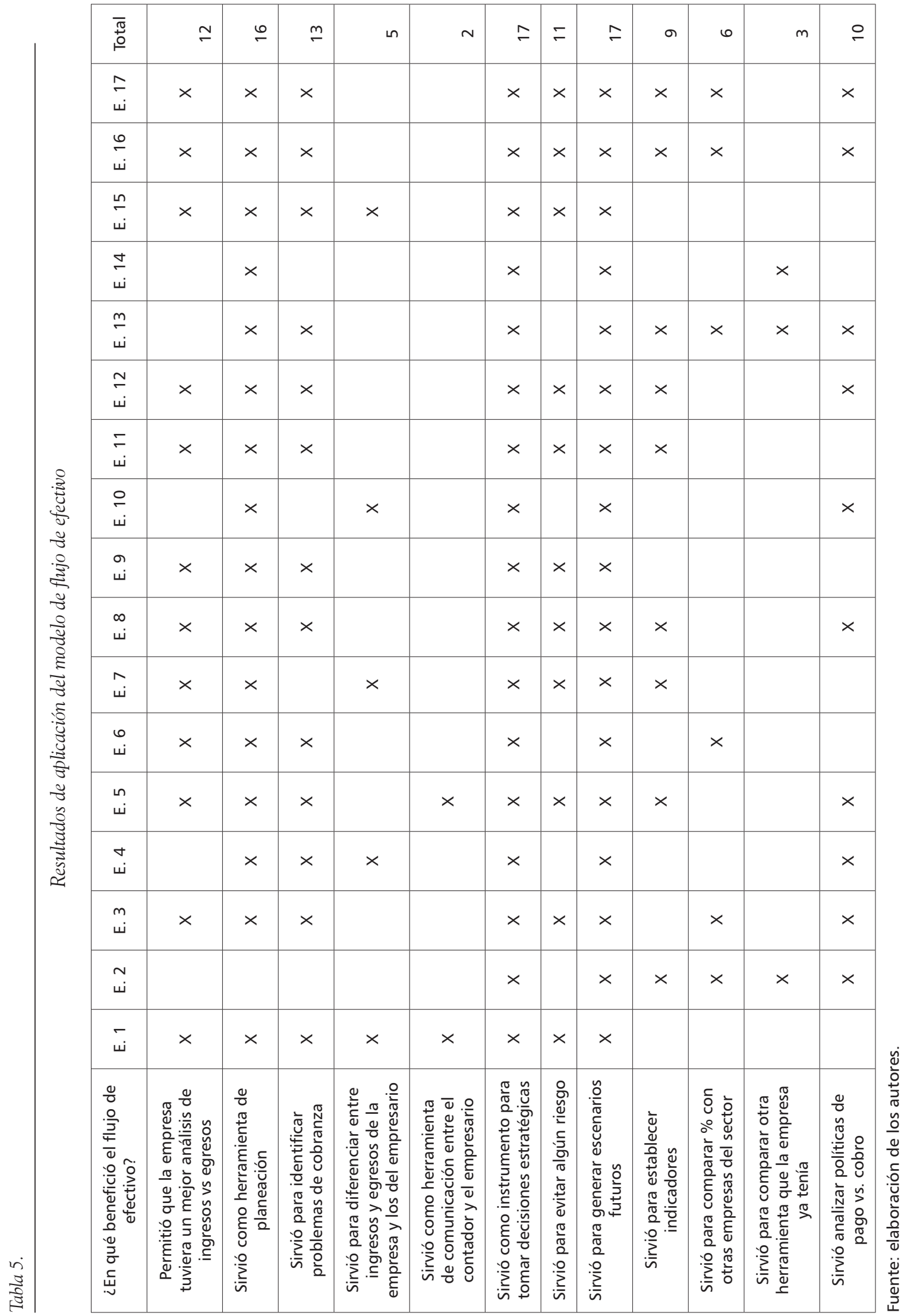


La tabla 5 refleja que entre los mayores impactos que se obtienen de esta metodología se encuentran su aplicación para tener un mayor análisis de ingresos y egresos de las pymes. En suma, es concebida como una herramienta que apoya la planeación, lleva a analizar y detectar problemas de cobranza, es un elemento importante en la toma de decisiones estratégicas, es útil para generar escenarios futuros y sirve en menor medida para analizar políticas de pago y cobro.

\section{CONCLUSIONES}

El flujo de efectivo es solo un elemento básico, aunque crítico, dentro de las pymes mexicanas; por ello, hacer análisis profundo de sus problemáticas, restricciones, proyecciones y estrategias permitirá a los directores de las empresas tomar decisiones mucho más claras para el desarrollo de su organización, y con ello, del tejido empresarial que está a su alrededor. El modelo de flujo de efectivo propuesto es una herramienta de gran utilidad, dado que se ha elaborado de acuerdo con las necesidades y especificaciones de los empresarios; su valor radica en que los rubros contenidos allí fueron resultado de las interacciones con el empresario y están adaptados a su proceso específico, de modo tal que se convierte en un "traje a la medida" y una herramienta que él maneja y comprende al haber sido partícipe de su construcción e implementación. Y, lo más importante: se puede adaptar a cualquier sector empresarial pyme.

Durante el desarrollo de este trabajo se encontró lo siguiente:

- La proyección del flujo de efectivo debe extenderse a un horizonte de 1 año con detalle mensual, y los siguientes 1-2 años, por cuatrimestres.

- La utilidad de la herramienta no está en función del consultor que la aplica, sino en la empresa que la utiliza como suya y que detalla los números para un análisis más detallado.
- Dentro de las aplicaciones se notó que otra de las problemáticas por analizar - $\mathrm{e}$ inclusive sería muy oportuno diseñar-es la de otra herramienta sencilla para generar indicadores de cobro. La cobranza es otro de los grandes problemas de las pymes; pero, como se ha mencionado, no es solo el hecho de no saber cobrar, sino no tener una estrategia definida y un mecanismo que permita al empresario tomar decisiones claras y permanentemente actualizadas. Y, aunque como señala Muscettola (2014), el crédito puede ser utilizado como una estrategia para mejorar la rentabilidad, un ciclo muy largo de cobranza impacta directamente al ciclo de conversión de efectivo, lo cual tiene un efecto negativo en la rentabilidad, en sintonía con lo evidenciado por Konuk y Zeren (2014).

- Muchos de los empresarios de mipymes no tienen un sueldo definido o asignado, lo cual es un indicador claro de la madurez o no de la empresa; no cobrar un salario implica que al frente se encuentra un emprendedor, y no un empresario. El empresario debe entender que la organización que creó o en la que está participando es un negocio y que es importante que tenga una remuneración clara por el trabajo que está desempeñando. En muchos casos, el empresario mezcla su dinero con lo que genera la empresa, sin tener un control, lo cual lleva a tener un empresario rico con empresa pobre. Por ello, él debe evaluar su flujo de efectivo y establecer un salario; es fundamental evitar que se mezcle el dinero del empresario con el de la empresa.

- Otro de los puntos clave es el excedente de flujo de efectivo y no saber qué hacer con él. En los casos en los que las empresas contaban con un flujo de efectivo sano y sin problemas en corto plazo, la problemática se centraba en el futuro. 
Al empresario mexicano le cuesta mucho la planeación y generar escenarios a mediano y largo plazo. Esta metodología permitió que los empresarios "jugaran" con posibilidades en la toma de decisiones, con miras a tomar iniciativas como invertir en áreas de innovación, investigación y desarrollo (esto, de hecho, es un aspecto clave para las pymes mexicanas). Lo menos favorable que puede hacer un empresario es tener efectivo $y$ no saber cómo invertirlo para que genere ingresos en el corto o largo plazo.

- Cuando su empresa está naciendo -y esta es pequeña o micro-, el empresario mexicano es un "todólogo", un "hombre orquesta", que va desde generar el producto o servicio, hasta vender, cobrar y atender problemáticas en el proceso. Este es un mal común que se encontró en las empresas analizadas; por ello, la metodología pretende que los empresarios también creen conciencia de que necesitan delegar actividades, dado que son claves para la permanencia de la empresa.

- La metodología sirve muy bien para mostrar las fallas que la empresa tiene y que el empresario, por estar dedicado a la operación, no ve con claridad. Se encontraron casos en los que se tenía conciencia de que las facturas de varios meses estaban por cobrar, pero no de la importancia de hacerlo efectivo y de toda la cartera vencida. Esta herramienta ayuda perfectamente a que el empresario entienda cómo está su ciclo de venta y cobro. Muchos empresarios se quedan en el vender bien, pero cuando tienen que pagar, no entienden por qué no cuentan con el recurso necesario para cubrir con sus obligaciones. Esto normalmente se ve claramente expresado en la falta de cobro.
- Es muy común que los empresarios usen el crédito bancario como una solución de emergencia a corto plazo; sin embargo, no se dan cuenta de que a largo plazo esto representa un problema serio en el flujo, ya que no pueden pagarse con facilidad los montos solicitados, y en el peor de los casos, se tiene que recurrir a otro tipo de financiamientos para cubrir los pagos del primero; se configura así un círculo vicioso del que la empresa no puede salir tan fácil.

Como se ha mencionado, la intención de este trabajo es generar una herramienta estratégica, mas no contable; es decir, el valor de esta metodología está en la estrategia, en el análisis detallado de las oportunidades internas y externas y en la generación de cultura ante el empresario y sus colaboradores. Los hallazgos permiten saber que existen una serie de anomalías en la administración del efectivo propias de las empresas de tamaño micro y pequeñas; y la principal razón de ello es la falta de una herramienta que, de acuerdo con necesidades específicas y elaborada por el propio empresario, permita controlar todas las operaciones de la empresa y brinde una visión clara del proceso de entradas y salidas de efectivo, así como de todas sus implicaciones.

La recomendación más importante es tratar de que la herramienta sea lo más amigable para el empresario, dado que él no contempla llevar ningún control de este tipo por considerarlo complejo; pero al comprender que puede ser adaptada a sus necesidades y ser más entendible, reconoce la utilidad y accede adoptarla como instrumento indispensable para controlar su flujo de efectivo. De este modo, cuenta con una herramienta fundamental en la toma de decisiones acerca de los sobrantes o faltantes de efectivo. Una última recomendación es que el empresario debe buscar capacitarse constantemente para que sea capaz no solo de elaborar el flujo, sino también de analizarlo y comprender sus implicaciones, dando seguimiento a las decisiones que tome con base en ello. 


\section{REFERENCIAS}

1. Ahmad, M. y Raza, K. (2012). The optimal relationship of cash conversion cycle with firm sizze and profitability. International Journal of Academic Research in Business and Social Sciences, 2(4), 189-203.

2. Bangs, D. (1998). The business planning guide. Chicago: Kapan Publishing.

3. Bolek, M., Kacprzyk, M. y Wolski, R. (2015). The relationship between value economic added and cash conversion cycle in companies listed in the WSE. Financial Internet Quartely Efinance, 8(2) 1-10.

4. Dakros, K. y Kallandranis, C. (2007). Investment and cash flow: Evidence for asymmetries in European for manufacturing. Applied Financial Economics, 17, 1191-1200.

5. Ebben, J. y Jonhson, A. (2011). Cash conversion cicle management in small firms: Relationships with liquidity, invested capital, and firm performance. Journal of Small Business Ë Entrepreneurship, 24(3), 381-396.

6. Faris, A. y Nassem, A. (2013). The relationship between cash conversion cycle and financial characteristics of industrial sectors: An empirical study. Investment Management and Financial Innovations, 10(4) 95-102.

7. Konuk, F. y Zeren, F. (2014). Is cash conversion cicles optimun in Turkish listed food beverages firms. Theorical and Apllied Economics, 21 (12), 153-164.

8. Kroes, J. y Manikas, A. (2014). Cash flow management manufacturing firm financial perfomance: A longitudinal perspective. Boise: Boise State University.

9. Loscalzo, W. (1994). Presupuesto de flujo de efectivo. Ciudad de México: Limusa.

10. Lyroudi, K. y McCarty, D. (1993). An empirical investigation of the cash conversion cycle of small business firms. The Journal of Entrepreneurial Finance, 2(2) 139-161.

11. Manoori, E. y Muhammad, D. (2012). Determinants of working capital management: Case of Singapore firms. Research Journal of Finance of Accountig, 3, 15-23.

12. Muscettola, M. (2014). Cash conversion cycle and firm's profitability: An empirical analysis on a Sample of 4,226 Manufacturing SMEs of Italy. International Journal of Business and Management, 9(5) 25-35.

13. Opiela, N. (2006). Keeping small-business cash flow on track. Journal of Financial Planning, 19(7), 26-32.

14. Owolabi, S. y Obida, S. (2012). Liquidity management and corporate profitability: Case study of selected manufacturing companies listed on the Nigerian stock exchange. Business Management Dynamics, 2(2), $10-25$.

15. Palacios, J., Flores, E. y García, A. (2013). Diagnóstico del sector TIC en México. Ciudad de México: Banco Interamericano de Desarrollo (BID).

16. ProMéxico (2015a). Sector automotriz. Recuperado de http://mim.promexico.gob.mx/wb/mim/ auto_perfil_del_sector

17. ProMéxico (2015b). Tecnologías de información. Recuperado de http://www.promexico.gob.mx/documentos/sectores/tecnologias-informacion.pdf

18. ProMéxico (2016). Sector automotriz. Situación actual: retos y oportunidades. Ciudad de México: Autor.

19. Saavedra, M. (2011). Herramientas de planeación financiera en las PYME. Ciudad de México: Gasca Sicco.

20. Secretaría de Economía de México (2009, 30 de junio). Acuerdo por el que se establece la estratificación de las micro, pequeñas y medianas empresas. Recuperado de http://dof.gob.mx/nota_detalle.php?codigo $=5096849 \&$ fecha $=30 / 06 / 2009$ 
21. Vargas, R. (2007). Estado de flujo de efectivo. Inter Sedes, 8(14) 111-136.

22. Yasir, M., Majid, A. y Yosuaf, Z. (2014). Cash conversion cycle and its impact upon firm perfomance: Evidence from cement industry of Pakistan. Global Business and Management Research: An International Journal, 6(2), 139-149.

23. Yin, R. K. (2003). Case study research: Design and methods (3. $\left.{ }^{\mathrm{a}} \mathrm{ed}.\right)$. Thousand Oaks: Sage. 